\title{
Decreased plasma tryptophan associated with deep white matter lesions in elderly subjects
}

\author{
H Yao, T Yuzuriha, H Koga, K Fukuda, K Endo, T Matsumoto, A Kato, A Uchino, \\ T Ezaki, S Ibayashi, H Uchimura, M Fujishima
}

Center for Emotional and Behavioral

Disorders, Hizen

National Hospital,

Saga, Japan

T Yuzuriha

H Koga

K Fukuda

K Endo

T Matsumoto

H Uchimura

Department of

Radiology, Saga

Medical School, Saga,

Japan

A Kato

A Uchino

Saga Central Health

Center

T Ezaki

Second Department of Internal Medicine,

Kyushu University,

Fukuoka, Japan

H Yao

S Ibayashi

M Fujishima

Correspondence to: Dr Hiroshi Yao, Second Department of Internal

Medicine, Faculty of

Medicine, Kyushu

University, Maidashi 3-1-1,

Higashi-ku, Fukuoka

812-8582, Japan. Telephone

008192642 5256; fax 0081

926425271 .

Received 27 April 1998 and in revised form

22 June 1998

Accepted 29 June 1998

\begin{abstract}
The aim was to identify potentially treatable risk factors for cerebral white matter lesions often found on MRI in elderly persons. findings were assessed on 1.0 T MRI of 178 subjects living in the community and aged 60 years or older. Participants underwent standardised evaluations including standard questionnaires, a physical and neurological examination, cognitive function tests, electrocardiogram, a complete blood chemistry panel, and plasma amino acid measurements. Brain MRI infarcts, deep white matter lesions (DWMLs), and periventricular hyperintensities were found in $26 \%, 43 \%$, and $29 \%$ of the 178 participants, respectively. Subjects with DWMLs were significantly older and had a higher frequency of hypertension, higher systolic blood pressure, and more brain infarcts, but lower plasma concentrations of tryptophan. In the multivariate model, greater age and lower plasma tryptophan concentrations were independently associated with DWMLs. Tryptophan concentrations were inversely related to DWML grading, whereas hypertension and brain infarction were more common in subjects with higher extents of DWMLs. The present study suggests that greater age and lower plasma tryptophan concentrations were important in producing DWMLs in elderly subjects.

(F Neurol Neurosurg Psychiatry 1999;66:100-103)
\end{abstract}

Keywords: magnetic resonance imaging; aging; amino acids

During the past decade, there has been mounting evidence that white matter hyperintensities on MRI are related to cognitive impairment, ${ }^{1-3}$ mood disorders, ${ }^{4-6}$ and gait disturbance or disequilibrium. ${ }^{7}$ Despite the fact that such deep white matter lesions (DWMLs) or leukoaraiosis often exist in healthy elderly subjects, only a few studies have focused on the pathogenetic mechanism for DWMLs. ${ }^{8}$ Recently, a large scale epidemiological study - the Cardiovascular Health Study-disclosed that white matter lesions were strongly related to age, silent stroke, hypertension, smoking history, and income. ${ }^{10}$ Among these variables, age had the strongest association with the grade of white matter lesions.

Oligodendroglia, which are a major cell type of white matter, were highly vulnerable to glutamate induced cell injury. Toxicity of glutamate to oligodendroglia is mediated through $\alpha$-amino-3-hydroxy-5-methyl-4-isoxazole propionic acid (AMPA)/kainate receptor activation (excitotoxicity), ${ }^{11}$ or by depletion of intracellular cystine and subsequent depletion of an antioxidant, glutathione. ${ }^{12}$ Increased blood brain barrier permeability, ${ }^{13}$ found in vascular dementia, could lead to an increased penetration of substances from blood to brain and could result in excitotoxic damage of oligodendroglia. Therefore, we determined the blood concentrations of glutamate and several related amino acids in elderly volunteers together with the MRI study.

\section{Subjects and methods}

Between June and August 1997, we examined 178 volunteers aged 61 to 91 years ( 37 men and 141 women with a mean age of 76.7 (SD 5.7) years), living in the rural community of Sefuri village, Saga, Japan (the total population of the village numbered 1980 at the time of examination). All subjects were living independently at home without apparent dementia. Years of education for the sample averaged 8.2 (SD 2.0) years. All subjects underwent a structured clinical interview. For the neurological examination, the presence of a Barré sign, asymmetric deep tendon reflexes, and Babinski's sign were recorded on each side. Tests of cognitive function included the Hasegawa dementia scale revised version (HDS-R),${ }^{14}$ and the mini mental state examination (MMSE). ${ }^{15}$ Scores of HDS-R and MMSE were linearly and significantly correlated $\quad(r=0.786$, $\mathrm{p}<0.001$ ). Based on the distributions and mean values of the scores of HDS-R and MMSE (25.7 (SD 3.5) and 25.6 (SD 3.5) respectively), decline in mental function was arbitrarily defined as HDS-R+MMSE $\leqslant 50$. Blood pressure was measured in the sitting position by the standard cuff method after a 5 minute rest. Arterial hypertension was considered present if a subject had a history of repeated high blood pressure recordings $>160 / 95 \mathrm{~mm}$ $\mathrm{Hg}$ or the subject was being treated for hypertension. Left ventricular hypertrophy 
Distribution of characteristics of study population with or without deep white matter lesions (DWMLs)

\begin{tabular}{|c|c|c|c|}
\hline & $\begin{array}{l}\text { DWMLs (+) } \\
(n=77)\end{array}$ & $\begin{array}{l}\text { DWMLs (-) } \\
(n=101)\end{array}$ & $p$ Value \\
\hline Age (y) & $78.1(5.8)$ & $75.6 \pm 5.5$ & 0.003 \\
\hline $\operatorname{Sex}(M / F)$ & $15 / 62$ & $22 / 79$ & NS \\
\hline Hypertension (+) & $37(48 \%)$ & $32(32 \%)$ & 0.026 \\
\hline $\mathrm{SBP}(\mathrm{mm} \mathrm{Hg})$ & $155(25)$ & $148(22)$ & 0.042 \\
\hline $\mathrm{DBP}(\mathrm{mm} \mathrm{Hg})$ & $77(10)$ & $74(10)$ & 0.052 \\
\hline Diabetes mellitus (+) & $4(5 \%)$ & $4(5 \%)$ & NS \\
\hline Hyperlipidaemia (+) & $19(25 \%)$ & $21(21 \%)$ & NS \\
\hline Heart disease $(+)$ & $5(6 \%)$ & $12(12 \%)$ & NS \\
\hline Abnormal ECG* ${ }^{\star}(+)$ & $14(18 \%)$ & $25(25 \%)$ & NS \\
\hline Alcohol consumption (+) & $15(19 \%)$ & $16(16 \%)$ & NS \\
\hline Smoking (+) & $8(10 \%)$ & $9(9 \%)$ & NS \\
\hline History of stroke (+) & $6(8 \%)$ & $2(2 \%)$ & NS \\
\hline Focal signs $(+)$ & $4(5 \%)$ & $8(8 \%)$ & NS \\
\hline Gait disturbance (+) & $2(3 \%)$ & $2(2 \%)$ & NS \\
\hline Brain infarction $(+)$ & $27(35 \%)$ & $21(21 \%)$ & 0.034 \\
\hline (No per case) & $2.15(1.94)$ & $1.24(0.63)$ & 0.044 \\
\hline \multicolumn{4}{|l|}{ Blood chemistry: } \\
\hline Packed cell volume & $0.37(0.04)$ & $0.37(0.04)$ & NS \\
\hline Albumin $(\mathrm{g} / \mathrm{l})$ & $42.1(3.2)$ & $42.8(2.5)$ & NS \\
\hline Fasting blood glucose $(\mathrm{mmol} / \mathrm{l})$ & $5.13(0.56)$ & $5.00(0.53)$ & NS \\
\hline Total cholesterol $(\mathrm{mmol} / \mathrm{l})$ & $5.08(0.87)$ & $4.98(0.94)$ & NS \\
\hline HDL cholesterol (mmol/1) & $1.52(0.33)$ & $1.46(0.39)$ & NS \\
\hline Creatinine $(\mu \mathrm{mol} / \mathrm{l})$ & $63.6(14.1)$ & $62.8(15.9)$ & NS \\
\hline Uric acid $(\mu \mathrm{mol} / \mathrm{l})$ & $291(88)$ & $272(81)$ & NS \\
\hline \multicolumn{4}{|l|}{ Amino acids: } \\
\hline Tryptophan $(\mu \mathrm{mol} / \mathrm{l})$ & $53.7(10.9)$ & $58.3(9.1)$ & 0.003 \\
\hline Tyrosine $(\mu \mathrm{mol} / \mathrm{l})$ & $62.0(10.8)$ & $65.2(11.7)$ & 0.067 \\
\hline Phenylalanine $(\mu \mathrm{mol} / \mathrm{l})$ & $56.3(9.3)$ & $56.4(7.3)$ & NS \\
\hline Valine $(\mu \mathrm{mol} / \mathrm{l})$ & $178.60(32.10)$ & $183.40(29.60)$ & NS \\
\hline Isoleucine $(\mu \mathrm{mol} / \mathrm{l})$ & $48.50(8.80)$ & $49.80(10.20)$ & NS \\
\hline Leucine $(\mu \mathrm{mol} / \mathrm{l})$ & $94.50(17.90)$ & $96.70(18.20)$ & NS \\
\hline Glutamic acid $(\mu \mathrm{mol} / \mathrm{l})$ & $31.5(10.6)$ & $31.5(10.8)$ & NS \\
\hline Glutamine $(\mu \mathrm{mol} / \mathrm{l})$ & $610.4(74.2)$ & $615.0(72.1)$ & NS \\
\hline Glycine $(\mu \mathrm{mol} / \mathrm{l})$ & $235.7(70.10)$ & $235.6(59.9)$ & NS \\
\hline Arginine $(\mu \mathrm{mol} / \mathrm{l})$ & $81.1(16.3)$ & $81.0(14.8)$ & NS \\
\hline Taurine $(\mu \mathrm{mol} / 1)$ & $55.4(14.8)$ & $53.0(10.3)$ & NS \\
\hline
\end{tabular}

$\mathrm{SBP}=$ Systolic blood pressure; $\mathrm{DBP}=$ diastolic blood pressure; $\mathrm{DWML}=$ deep white matter lesion; values are mean $(\mathrm{SD}) ; \mathrm{NS}=$ not significant $(\mathrm{p}>0.1)$.

${ }^{\star}$ Left ventricular hypertrophy, ischaemic change, or atrial fibrillation.

(Minnesota code 3-3-2), ST depression (4-1), or atrial fibrillation (8-3-2) were registered on 12 lead ECG recordings. Diabetes mellitus was defined as a fasting plasma glucose greater than $7.77 \mathrm{mmol} / 1$ or a previous diagnosis of diabetes mellitus. Hyperlipidaemia was defined as total serum cholesterol concentration $>5.69 \mathrm{mmol} / 1$ or if the subject was being treated for hyperlipidaemia at the time of examination. Plasma concentrations of 11 amino acids as shown in the table were determined with the standard fluorimetric HPLC-OPA method. ${ }^{16}$

All MRI was performed on a $1.0 \mathrm{~T}$ superconducting magnet (MAGNEX $\alpha$, Shimadzu, Kyoto, Japan) using the spin echo technique and fluid attenuated inversion recovery (FLAIR) sequences. Transverse T1 weighted (TR/TE $380 / 14 \mathrm{~ms}$ ), T2 weighted (TR/TE 3750/110 ms), and FLAIR (TR/ TI/TE 5800/1700/110 ms) images were obtained with a slice thickness of $8 \mathrm{~mm}$ separated by a $2 \mathrm{~mm}$ interscan gap. All scans were reviewed by two independent investigators ( $\mathrm{HY}$ and $\mathrm{AK}$ ) who were blinded to all clinical data. Brain infarcts were defined as lesions with abnormal signal in a vascular distribution and no mass effects, and were defined as low signal intensity areas on $\mathrm{T} 1$ weighted images and high signal intensity areas on T2 weighted images and their sizes were more than $5 \mathrm{~mm}$. Deep white matter lesions (DWMLs) and periventricular hyperintensities (PVHs) were specified as high signal intensity areas on T2 weighted images but isointense with normal brain parenchyma on $\mathrm{T} 1$ weighted images. The severity of deep white matter lesions was graded according to Fazekas $e a^{17}{ }^{18}$ as follows; grade 0, absent; grade 1, punctate; grade 2, beginning confluent; grade 3 , large confluent.

All values are given as mean (SD). We used the SPSS software (SPSS Advanced Statistics $^{\mathrm{TM}}$ version 6.1, SPSS Inc, Chicago, IL, USA) for statistical analysis. Univariate analyses were performed with a $t$ test for continuous variables and $\chi^{2}$ test for categorical variables. Multivariate analyses were done with a logistic regression model to identify factors predictive of cognitive decline or DWMLs. We chose variables for entry of multivariate analyses on the basis of clinical importance from those with values of $p<0.1$ after univariate testing. For multiple comparisons, an analysis of variance followed by unpaired $t$ test was used. The Bonferroni principle was used to correct for multiple comparisons, with the level of significance set at $p<0.0167$. Pearson's correlation coefficient was used for linear regression procedure.

\section{Results}

Hypertension was present in 69 subjects. Mean blood pressure levels were $144(21) / 73$ (10) $(\mathrm{n}=109), 161(22) / 78(11)(\mathrm{n}=63)$, and 184 (25)/88 (7) $(\mathrm{n}=6) \mathrm{mm} \mathrm{Hg}$ in normotensive subjects, treated hypertensive subjects, and non-treated hypertensive subjects respectively. The prevalences of diabetes mellitus, hyperlipidaemia, and heart disease were $4 \%, 22 \%$, and $10 \%$ respectively. History of minor stroke or gait disturbance was found in eight cases. Infarction was detected by MRI in 48 of the 178 participants $(26 \%)$. Small and deeply situated lacunar infarcts were the predominant type. These infarcts were asymptomatic in 43 of the 48 subjects (90\%); DWMLs and PVHs were present in $77(43 \%)$ and $51(29 \%)$ subjects, respectively.

In multivariate analysis of 176 participants undergoing HDS- $\mathrm{R}$ and MMSE, age $(p=0.030)$, education $(p=0.009)$, and DWMLs $(p=0.028)$ were independently associated with cognitive decline. Univariate analyses disclosed that DWMLs were associated with increased age, hypertension, systolic blood pressure, brain infarction, and the lower blood concentration of tryptophan (table). Among the variables age, hypertension, brain infarction, and tryptophan, age $(\mathrm{p}=0.0333)$ and tryptophan $(p=0.0095)$ were significant factors concerning DWMLs, whereas hypertension was not significant $(\mathrm{p}=0.1064)$ by a multiple logistic regression analysis. Even if tyrosine (non-significant decrease in DWML (+) group) was forced into the multivariate model, tryptophan and age were still independently associated with DWMLs. No linear relation existed between age and plasma tryptophan concentrations in subjects with and without DWMLs. As shown in the figure, plasma tryptophan concentrations were inversely correlated with the DWML grading. Subjects with grade 2 DWMLs were older than those without DWMLs. Hypertension and brain infarction were more prevalent in those with grades 2 and 3 DWMLs. 


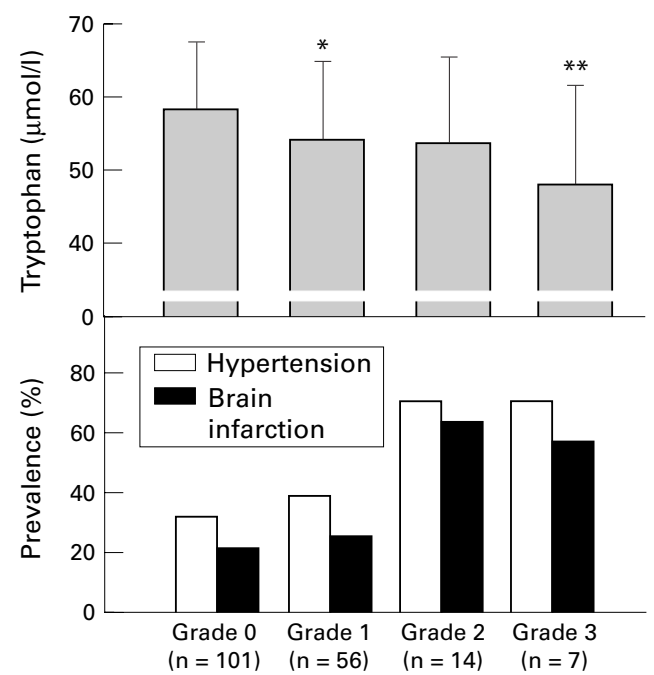

Plasma tryptophan concentrations were inversely correlated with the DWML grading: grade 0, 58.3 (9.1); grade 1, 54.4 (10.4); grade $2,53.7$ (11.8); grade 3, 48.2 (13.50) umol/l $\left({ }^{\star} p<0.0167,{ }^{\star *} p<0.01\right.$ v grade 0 , analysis of. variance (ANOVA) and unpaired t test). Hypertension and brain infarction were more common in grades $2-3$ DWMLs than in those with grade 0-1 DWMLs: the prevalences of hypertension were $71 \%$ v 34\% ( $p=0.0011$, $\chi^{2}$ test) and those of brain infarction were $62 \%$ v $22 \%$ ( $p=0.0001)$ in grades $2-3$ DWMLs and grades $0-1$ DWMLs, respectively.

\section{Discussion}

We found a significant association of DWMLs and blood concentrations of tryptophan even after adjustment for age, hypertension, and brain infarction. A clear inverse relation existed between the DWML grading and plasma tryptophan concentrations. The importance of vascular risk factors was confirmed by recent compelling evidence obtained from MRI of 3301 elderly participants in the Cardiovascular Health Study. ${ }^{10}$ In addition to the classic vascular risk factors, thrombogenic and haemostatic factors (raised fibrinogen and increased factor VIIc concentrations $)^{9}$ and decreased vitamin $\mathrm{E}^{8}$ were associated with DWMLs. Our new finding was that decreased tryptophan was associated with DWMLs.

Tryptophan is a large neutral amino acid which is utilised in the biosynthesis of various substances such as serotonin and melatonin. These indoleamines act as antioxidants. ${ }^{19}$ In this context, our results accord well with those by Schmidt et al. ${ }^{8}$ which provided evidence of an association between low plasma concentrations of vitamin $\mathrm{E}$ and a high risk of cerebral white matter lesions. Niacin or nicotinic acid is also synthesised from tryptophan; a dietary deficiency in tryptophan and niacin can lead to pellagra. Experimental nicotinamide deficiency produced by an analogue of the vitamin 6-aminonicotinamide caused more toxicity toward glial elements than neurons. ${ }^{20}{ }^{21}$ Interestingly, the earliest histological change noted was a mild swelling of glial cells, in particular oligodendroglia. ${ }^{21}$ The rate limiting enzyme in serotonin synthesis, tryptophan hydroxylase, is normally unsaturated, so serotonin concentrations are determined by free tryptophan. Serotonin protects C6 glioma cells from excitotoxicity induced by $10 \mathrm{mM}$ of glutamate. ${ }^{22}$ The protective action of serotonin was considered to be mediated through the 5HT1A type of serotonin receptors. However, no available data so far have found a protective action of serotonin against oligodendroglia injury or DWMLs. Further studies are needed to elucidate the possible involvements of oxidative damage or niacin deficiency, and the protective role of serotonin in DWMLs.

Brain infarction on MRI was not strongly related with DWMLs in the present study. This negative result does not exclude the possibility that the pathophysiology of the two conditions (brain infarction and DWMLs) may be similar. If risk factors have a declining impact with increasing age, the causal factor for DWML may be different from major accepted risk factors for arteriosclerosis in our study population with considerably advanced age. Furthermore, $91 \%$ of hypertensives received antihypertensive treatment, so the effects of hypertension, which underlie the risk of brain infarction, could be attenuated in our sample. However, as shown in the figure, confluent DWMLs were often associated with hypertension and brain infarction. In line with our findings, studies on pathological correlates of incidental white matter lesions on MRI disclosed that confluent DWMLs consisted of incomplete parenchymal destruction with focal transitions to infarcts, whereas punctate DWMLs were not associated with infarction. ${ }^{18}$ Taken together, aging and lower tryptophan concentrations are tentatively assumed to be factors which are essentially linked to DWMLs in our study population, and classic vascular risk factors may accelerate or exacerbate the process of white matter "ischaemic" damage.

The present study showed that DWMLs seen in elderly subjects was related to age and lower blood tryptophan concentrations. Because the present results were obtained from a cross sectional survey, inferences regarding a causal relation between DWMLs and tryptophan remain tentative. Longitudinal studies will be needed to determine whether such possibly treatable factors as lower plasma concentrations of tryptophan contribute to the evolution of DWMLs.

We gratefully acknowledge N Kawahara, T Hirotaki, C Morita, O Mashima, and M Yamaguchi for registration of participants, Dr T Nakahara, and Dr K Nakamura for their valuable advice during the course of this study, K Sato, Y Oda, M Nakano, and $\mathrm{K}$ Watanabe for the assessments of cognitive function, and $\mathrm{T}$ Muto and T Wada for laboratory assays. We also thank August McInnis for editing the manuscript. This study was partly supported by Grants in Aid for Scientific Research from Sasagawa Health Science Foundation and the Ministry of Health and Welfare in Japan.

1 Boone KB, Miller BL, Lesser IM, et al. Neuropsychological correlates of white-matter lesions in healthy elderly subjects. A threshold effect. Arch Neurol 1992;49:549-54.

2 Schmidt R, Fazekas F, Offenbacher H, et al. Neuropsychologic correlates of MRI white matter hyperintensities: a study of 150 normal volunteers. Neurology 1993;43:24904.

3 Breteler MMB, van Amerongen NM, van Swieten JC, et al. Cognitive correlates of ventricular enlargement and cerebral white matter lesions on magnetic resonance imaging. The Rotterdam Study Stroke 1994;25:1109-15.

4 Coffey CE, Figiel GS, Djang WT, et al. Subcortical hyperintensity on magnetic resonance imaging: a comparison of tensity on magnetic resonance imaging: a comparison of
normal and depressed elderly subjects. Am $\mathcal{F}$ Psychiatry normal and depress 
5 Zubenko GS, Sullivan P, Nelson JP, et al. Brain imaging Zubenko GS, Sullivan $\mathrm{P}$, Nelson abnormalities in mer

6 Dupont RM, Jernigan TL, Heindel W, et al. Magnetic resonance imaging and mood disorders. Localization of white matter and other subcortical abnormalities. Arch Gen Psychiatry 1995;52:747-55.

7 Baloh RW, Yue Q, Socotch TM, et al. White matter lesions and disequilibrium in older people. I. case-control comparison. Arch Neurol 1995;52:970-4.

8 Schmidt R, Hayn M, Fazekas F, et al. Magnetic resonance imaging white matter hyperintensities in clinically normal elderly individuals. Correlations with plasma concentrations of naturally occurring antioxidants. Stroke 1996;27:2043-7.

9 Breteler MMB, van Swieten JC, Bots ML, et al. Cerebral white matter lesions, vascular risk factors, and cognitive function in a population-based study: the Rotterdam Study. Neurology 1994;44:1246-52.

10 Longstreth WT, Manolio TA, Arnold A, et al, for the Cardiovascular Health Study collaborative Research Group. Clinical correlates of white matter findings on cranial magnetic cal correlates of white matter findings on cranial magnetic resonance imaging of 3301 elderly people. The

11 Yoshioka A, Hardy M, Youkin DP, et al. $\alpha$-amino-3-hydroxy5-methyl-4-isoxazolepropionate (AMPA) receptors mediate excitotoxicity in the oligodendroglial lineage. $f$ Neurochem 1995;64:2442-8

12 Oka A, Belliveau MJ, Rosenberg PA, et al. Vulnerability of oligodendroglia to glutamate: pharmacology, mechnism, and prevention. $\mathcal{F}$ Neurosci 1993;13:1441-53.
13 Wallin A, Blennow K, Fredman P, et al. Blood brain barrier function in vascular dementia. Acta Neurol Scand 1990;81: $318-22$

14 Katoh S, Simogaki H, Onodera A, et al. Development of the revised version of Hasegawa's dementia scale (HDS-R) (in Japanese). Ipn f Geriatr Psychiatry 1991;2:1339-47.

15 Folstein MF, Folstein SE, McHugh PR. Mini-mental state: a practical method for grading the cognitive state of patients for clinician. F Psychiatr Res 1975;12:189-98.

16 Deyl Z, Hyanek J, Horakova M. Profiling of amino acids in body fluids and tissues by means of liquid chromatography. f Chromatogr 1986;379:177-250

17 Fazekas F, Chawluk JB, Alavi A, et al. MR signal abnormalities at $1.5 \mathrm{~T}$ in Alzheimer's dementia and normal aging. AfNR Am f Neuroradiol 1987;8:421-26.

18 Fazekas F, Kleinert R, Offenbacher H, et al. Pathologic correlates of incidental MRI white matter signal hyperintensities. Neurology 1993;43:1683-9.

19 Politi V, D'Alessio S, Di Stazio G, et al. Antioxidant properties of indole-3-pyruvic acid. In: Filippini GA, et al. Recent ties of indole-3-pyruvic acid. In: Filippini GA, et al. Recent
advances in tryptophan research. New York: Plenum Press, advances in tryptop

20 Schneider H, Cervos-Navarro J. Acute gliopathy in spinal cord and brain stem induced by 6 -aminonicotinamide. Acta Neuropathol 1974;27:11-23.

21 Griffiths IR, Kelly PAT, Grome JJ. Glucose utilization in the central nervous system in the acute gliopathy due to 6-aminonicotinamide. Lab Invest 1981;44:547-52

22 Shinagawa S. Serotonin protects C6 glioma cells from glutamate toxicity. Neuroscience 1994;59:1043-50.

\section{NEUROLOGICAL STAMP}

\section{Theodore Schwann (1810-82)}

The German physiologist Theodore Schwann made one of the great biological discoveries when he founded modern histology by defining the cell as the basic unit. He has not been honoured philatelically but has been the subject of a German postal cancellation. While Professor of Anatomy and Physiology at Liege he described (1838) the myelin sheath as a fat-like substance which he considered to be a secondary deposit on the inner surface of a structural cell membrane (called neurilemma by Bichat). He also investigated muscular contraction and nerve structure, discovered muscle in the upper oesophagus, and coined the term metabolism for the chemical changes that take place in living tissue. $\mathrm{He}$ also formulated the basic principles of embryology and the role played by microorganisms in putrefaction.

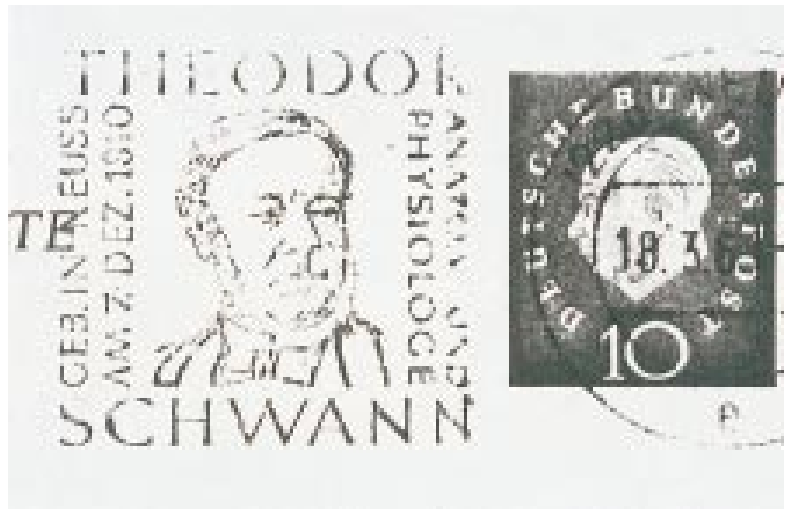

